\title{
L'aéroport à Raivavae (Australes) : développement économique, migrations et identités
}

\section{Gwendoline Malogne-Fer}

\section{Q OpenEdition \\ 12 Journals}

Édition électronique

URL : http://journals.openedition.org/jso/203

DOI : $10.4000 /$ jso.203

ISSN : $1760-7256$

Éditeur

Société des océanistes

Édition imprimée

Date de publication : 1 décembre 2004

Pagination : 189-199

ISSN : 0300-953x

\section{Référence électronique}

Gwendoline Malogne-Fer, «L'aéroport à Raivavae (Australes) : développement économique,

migrations et identités », Journal de la Société des Océanistes [En ligne], 119 | Année 2004-2, mis en ligne le 01 décembre 2007, consulté le 20 avril 2019. URL : http://journals.openedition.org/jso/203 DOI : $10.4000 /$ jso. 203

(c) Tous droits réservés 


\title{
L'aéroport à Raivavae (Australes) : développement économique, migrations et identités
}

par

\author{
Gwendoline MALOGNE-FER*
}

\section{RÉSUMÉ}

La construction d'aéroports comme celui de Raivavae (îles Australes) s'inscrit dans une politique de désenclavement des îles éloignées, en vue d'un développement " durable et équilibré ». Il vise aussi à combler un "écart", notion qui repose à la fois sur des critères objectifs - niveau de revenus monétaires, exportations agricoles, infrastructures - et sur une représentation idéalisée des îles dites " traditionnelles », que la modernité n'aurait pas encore atteintes. Dès lors, la décision prise en 1997 d'ouvrir un aéroport à Raivavae cristallise deux aspirations contradictoires : d'un côté, la préservation d'un environnement et d'une identité culturelle patrimonialisées; de l'autre, la diffusion du progrès jusqu'aux îles lointaines. Les Raivavae qui ont émigré à Tahiti sont particulièrement sensibles à cette contradiction. S'ils se réjouissent de la possibilité qui leur est désormais offerte de retourner plus facilement dans leur ile (alors que beaucoup ne l'ont plus revue depuis des décennies), ils s'inquiètent aussi de la dénaturation probable d'un mode de vie et d'une terre qui, dans leur mémoire, reste comme un espace hors du temps, miraculeusement préservé des évolutions auxquelles euxmêmes se sont habitués, depuis qu'ils vivent à Tahiti.

MoTS-CLÉS : aéroport, migrations, identité, mémoire, Polynésie française.

Depuis le début des années 1960 et l'implantation du Centre d'expérimentation du Pacifique

\section{ABSTRACT}

The erection of airports such as that at Raivavae (South Sea islands) is part of a policy geared towards opening up the outlying islands and planning a "lasting and well-balanced" development. It also aims to fill a "gap", a notion that is based on unbiased criteria - monetary incomes, agricultural export, infrastructures - as well as on an idealized representation of the so-called "traditional" islands that modernity has not reached. The decision taken in 1997 to open an airport in Raivavae crystallizes contradictory aspirations : on the one hand, the preservation of the environment and of a cultural identity belonging to the island's heritage and on the other the spreading of progress to the outlying islands. The natives of Raivavae who have moved to Tahiti are particularly sensitive to this contradiction. If they are delighted about the possibility of going back to their island easily (whereas many of them haven't seen it again in decades), they are also worried about the probable distortion of a way of living and of a land (fenua), which in their memories, remains a place out of time, miraculously saved from the evolutions they themselves have gotten used to since moving to Tahiti.

KeYwORDS: airport, migrations, identity, memory, French Polynesia.

(CEP) sur les atolls de Moruroa et de Fangataufa aux Tuamotu, le territoire de Polynésie française

* Sociologue, UMR CNRS-EHESS 8034, Centre d'étude interdisciplinaire du fait religieux (CEIFR), gmalogne@ehess.fr 
a connu d'importantes transformations sociales et économiques (emplois salariés, urbanisation, évolution des modes de consommation) associées à une évolution institutionnelle accordant à chaque nouveau statut une plus grande autonomie politique vis-à-vis de la métropole ( $\mathrm{Al}$ Wardi, 1998).

Mais le processus d'autonomie politique ne s'accompagne pas ipso facto d'une diminution des transferts de fonds publics de l'État vers la Polynésie française puisque les dépenses du CEP sont reconverties en dépenses civiles, grâce notamment au Fonds pour la reconversion économique de la Polynésie française (FREPF). L'aide annuelle pour la décennie 1996-2006 s'élève ainsi à dix-huit milliards de FCFP soit un milliard de FF (Poirine, 2002 : 326). L'autonomie financière de la Polynésie française signifie moins, dans ce contexte, un désengagement de l'État qu'une aide qui serait désormais allouée sous forme de subventions globales, à charge pour la Polynésie française d'utiliser ces subventions selon ses propres priorités.

Le moratoire sur les essais nucléaires en 1992 et l'arrêt définitif de ces derniers en 1995 ont incité l'État et le territoire de la Polynésie française à s'engager dans une dynamique de développement entendu comme une nouvelle phase destinée à accroître les ressources propres du territoire grâce, notamment, à la croissance de certains secteurs comme le tourisme, la perle et la pêche, un développement qui se doit d'être durable et harmonieux en associant l'ensemble des Polynésiens, y compris ceux des archipels éloignés (Tuamotu-Gambier, Australes et Marquises). C'est pourquoi le désenclavement des archipels est une des priorités du territoire, priorité rappelée dans le cadre du pacte de progrès de 1993 et, plus récemment, dans le cadre du contrat de développement 2000-2003 qui a pour objectif :

« l'accroissement durable et équilibré des ressources propres du territoire, tout en renforçant la cohésion sociale et l'identité culturelle de la population polynésienne. »

Il s'agit, dans cette perspective, de financer la construction d'infrastructures de communication modernes (routes, ports, aéroports) indispensables au développement économique des archipels.

L'ouverture, en novembre 2002, de la piste d'aviation à Raivavae', dans l'archipel des Aus- trales, est la concrétisation d'un projet relativement ancien qui s'inscrit dans le cadre de cette politique gouvernementale. À terme, l'ensemble des îles des Australes devrait être dotées d'aéroports puisque le projet est en cours de réalisation à Rimatara et en discussion à Rapa. Il s'agit tout à la fois de lutter contre la dépopulation qui touche cet archipel et la trop forte concentration de la population émigrée en zone urbaine. Désormais, Tahiti concentre $70 \%$ de la population de Polynésie française et un habitant sur deux vit en zone urbaine (ISTAT, 2003a : 4). En ce sens, le désenclavement des archipels est indissociable de la question de l'exode rural et l'aéroport de Raivavae concerne à la fois ceux qui y vivent et ceux qui ont émigré à Tahiti. Nous analyserons ici les enjeux et les retombées attendues de la piste d'aviation à Raivavae en adoptant comme axe privilégié la question des migrations et la reconfiguration des relations entre les communautés de Raivavae et Tahiti.

L'aéroport est habituellement présenté comme une " ouverture sur le monde », il s'inscrit ainsi dans une compréhension du développement en termes de dynamique extérieure à laquelle il convient de s'adapter ou de résister (1). Parce qu'il se situe à la frontière entre l'intérieur et l'extérieur de l'île, l'aéroport est un point de passage obligé des personnes, des biens et des idées, il remet en cause les modes traditionnels d'organisation et de représentation de la communauté insulaire (2). L'ouverture d'une piste d'aviation engendrant de fait une reconfiguration des relations entre les insulaires et les Raivavae émigrés à Tahiti, elle ravive les interrogations de ces derniers sur leur exil et leur propre conception de la modernité (3).

\section{Tahiti, Tubuai et Raivavae : un développement géographiquement différencié}

Composées de Raivavae, Rapa, Rimatara, Rurutu et Tubuai, les îles Australes ou Tuha'a pae au sud de Tahiti (cf. carte 1) totalisent, au recensement de 2002, 6386 habitants soit 2,6\% de la population de la Polynésie française (contre $6 \%$ en 1951). L'archipel est essentiellement tourné vers l'agriculture et, dans une moindre mesure, la pêche et l'artisanat. La population est en grande majorité protestante et pratiquante, à l'exception de l'île de Tubuai où la diversité confessionnelle est relativement ancienne (la

1. Cet article repose sur des entretiens effectués à Tahiti entre octobre 2000 et février 2002 auprès de personnes originaires de Raivavae et de Rapa, mais habitant Tahiti et fréquentant pour la grande partie d'entre eux (à des degrés divers) une paroisse protestante. Ces recherches s'inscrivent dans le cadre d'un projet d'études coordonné par l'anthropologue Jeanette DickersonPutman (université d'Indianapolis). 




CARTE 1. - Distances et modes de transport interinsulaires.

mission mormone est implantée à Tubuai depuis 1844). Les îles Australes se trouvent ainsi associées dans l'imaginaire tahitien à un mode de vie traditionnel, à la fois rural et protestant. Partant à Rurutu en 1980, Jean-Claude Guillebaud écrivait ainsi :

«À Tahiti, on rêve bruyamment à cet archipel éloigné au sud du Sud, pour les mêmes raisons qu’à Paris, on rêve de Tahiti. C'est le mythe à l'intérieur du mythe. C'est, m'a-t-on répété, un périmètre intact, un morceau de passé en état de marche, le conservatoire des nostalgies "écolos". » (Guillebaud, $1980:$ 50)

Mais, au sein de l'archipel, une nouvelle distinction s'opère selon les îles, entre celles qui disposent d'un aéroport (c'est le cas de Tubuai) et celles, comme Raivavae jusqu'en 2002, accessibles uniquement par bateau. Une comparaison rapide des deux îles permet de prendre la mesure des conséquences attendues de l'ouverture d'une piste d'aviation à Raivavae.

L'île de Tubuai (1979 habitants en 2002), située à $640 \mathrm{~km}$ au sud de Tahiti et à $200 \mathrm{~km}$ à l'est de Raivavae, est le centre administratif des Australes et concentre à ce titre un certain nombre de services administratifs (gendarmerie et trésorerie des Australes), hospitaliers (avec un médecin, un dentiste et deux infirmiers) et éducatifs : le collège scolarise en 1999-2000 quatre cents élèves (notamment ceux de Rapa et Raivavae) et emploie vingt-six personnes, enseignants, administratifs et techniciens. Parmi les activités économiques de l'île, le secteur agricole est dominant. Il s'agit d'un secteur diversifié et professionnalisé - comme l'atteste les tailles d'exploitation et les nombreuses sorties d'indivision ${ }^{2}$ tourné vers le secteur marchand. Les pommes de terre, carottes et choux verts sont commercialisés à Tahiti grâce aux liaisons maritimes assurées par le bateau Tuha'a pae deux à trois fois par mois. La production légumière atteignait ainsi 1400 tonnes en 1996 (ministère des Archipels, 1998 : 22). Les infrastructures touristiques restent relativement modestes.

Raivavae (995 habitants en 2002) située à $710 \mathrm{~km}$ de Papeete (cf. carte 2) est accessible, jusqu'en novembre 2002, uniquement par bateau. La population est répartie en trois villages : Rairua-Mahanatoa, le centre administratif, Vairua qui a été choisi pour accueillir la piste d'aviation et Anatonu. L'île possède un Groupement d'observation dispersé (GOD) qui scolarise une cinquantaine d'élèves en classes de sixième et cinquième. L'absence de structures éducatives après la cinquième oblige les élèves à partir à Tubuai ou Tahiti et cet exil forcé constitue aux yeux de nombreux parents une cause importante d'échec scolaire : les enfants sont « très pertur-

2. Contrairement à ce qu'affirme Bruno Saura (1995: 117-119), les sorties d'indivision ne sont pas liées à la construction de l'aéroport à Tubuai puisqu'elles lui sont antérieures. Michel Panoff note ainsi, à partir d'études ethnologiques effectuées entre 1961 et 1963, que « l'individualisme apparaît comme le trait dominant de la mise en valeur des terres à Tubuai » (1970 : 90$)$. 


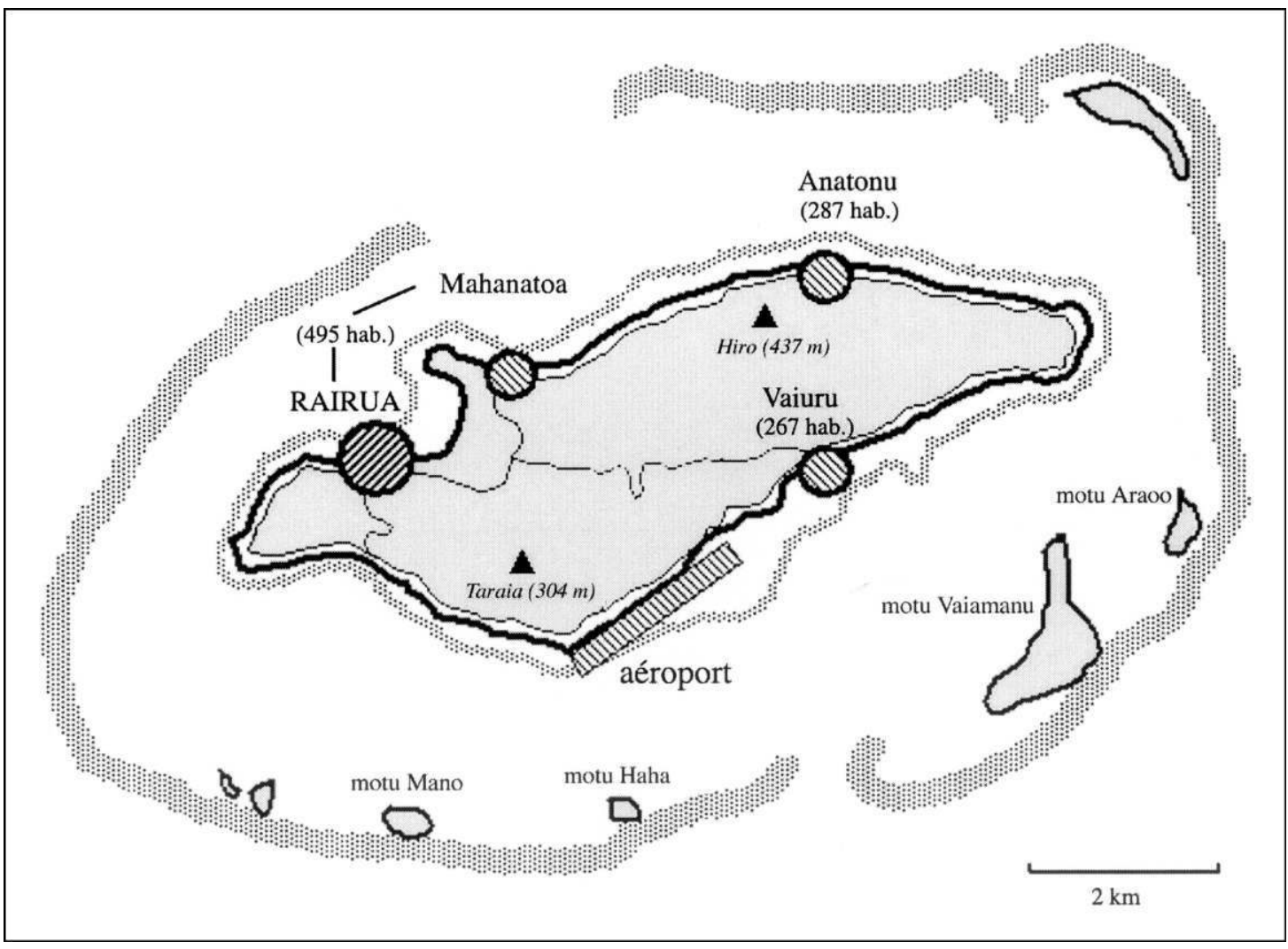

CARTE 2. - L'île de Raivavae.

bés, ils arrivent à Tubuai pas pour travailler, mais parce qu'il faut aller à Tubuai. Ils vont de surprises en surprises et après c'est l'échec ", dit un pasteur. L'absence de médecin et de dentiste oblige aussi à partir à Papeete pour se faire soigner. La production agricole commercialisée est beaucoup plus réduite qu'à Tubuai (13 tonnes en 1996).

La description comparée des îles de Tubuai et de Raivavae illustre la façon dont les transformations des modes de vie et de consommation depuis quarante ans affectent différemment la population selon les îles. La maîtrise de la langue française et la présence de Français et d'étrangers permettent de mesurer cette emprise différenciée de l'occidentalisation des modes de vie et de consommation. Alors qu'à Tahiti, 4,5\% de la population (âgée de 14 ans et plus) n'a aucune connaissance du français, ils sont $20 \%$ à Tubuai et $32 \%$ à Raivavae (ISTAT, $1996: 146-147$ ). Les métropolitains et étrangers représentent 15,5\% de la population à Tahiti, $6,5 \%$ à Tubuai et $3 \%$ à Raivavae. De même, la part de l'autoconsommation (qui correspond à la consommation de produits non achetés) permet de mesurer la façon dont le secteur marchand affecte inégalement la population polynésienne : la part non monétaire de la consommation alimentaire est ainsi de $57 \%$ aux Australes et de $25 \%$ à Tahiti en zone urbaine. Les modalités de professionnalisation de secteurs d'activités comme l'agriculture ou plus encore la pêche se heurtent de fait aux habitudes d'échanges non monétarisés dans le cadre de relations de parenté (Fer, 2000); la commercialisation à l'extérieur de l'île permet de rentabiliser l'exploitation, mais rend du même coût la dépendance à l'égard des moyens de transports particulièrement forte.

La compréhension de la modernité la plus communément admise est que les transformations sont d'abord initiées à Tahiti avant d'affecter les îles. Cette cartographie des évolutions en cours se nourrit d'un sentiment diffus d'être toujours « en retard » : ce sentiment se retrouve chez les instituteurs qui déplorent le retard de leurs élèves (qui travaillent à partir de manuels scolaires trop anciens) comme chez les hommes politiques (qui regrettent le retard pris en matière d'infrastructures et d'équipement). Pourtant, si certains taux d'équipements permettent de mesurer cet « écart »- les ménages sans automobile représentent $22 \%$ des ménages à Tahiti, $50 \%$ à Tubuai et $79 \%$ à Raivavae (ISTAT, 1996 : 184-185) -, d'autres secteurs comme celui de 
l'information et des communications soulignent au contraire un accès quasi égal quelle que soit l'île. Ainsi, depuis 1993, les Raivavae ont le téléphone et reçoivent les programmes de télévision de RFO - et plus récemment de Tahiti Nui TV - ; en $1996,78 \%$ des ménages ont la télévision, $61 \%$ la radio et $56 \%$ le téléphone (ISTAT, 1996 : 182-183). Surtout, le taux élevé d'endettement moyen des ménages aux Australes (ISTAT, 2003b : 113) laisse supposer qu'il existe un écart entre le mode de vie traditionnel et les aspirations de la population. De ce point de vue, l'ouverture d'une piste d'aviation à Raivavae peut être perçue comme un moyen de réduire cet écart et d'accéder à un mode de vie comparable à celui de Tahiti.

\section{L'aéroport à Raivavae : histoire d'une décision controversée et premières retombées}

Le projet d'aéroport à Raivavae a suscité dans un premier temps de vives réactions, mettant à jour la complexité des intérêts et des enjeux à la fois politiques, fonciers, sanitaires et économiques et l'imbrication des autorités religieuses et politiques dans le processus décisionnel.

Le projet initial d'un aéroport à Raivavae date des années 1970. Il était à l'époque situé sur un des nombreux îlots entourant l'île - elle-même trop montagneuse pour accueillir une piste. Et si, finalement, le projet a abouti en 1997 à l'initiative du maire, c'est d'abord parce que l'emplacement choisi, dans le lagon ${ }^{3}$, permettait d'éviter le recours aux procédures d'expropriation foncière particulièrement difficiles à mettre en ouvre lorsqu'il s'agit de terres indivises comme c'est le cas pour la majeure partie des terres de Raivavae.

Les travaux de la piste, d'une longueur de $1,3 \mathrm{~km}$, ont démarré fin 1999. Le chantier, dirigé par les services territoriaux de l'équipement et prévu pour une durée initiale de dix-huit mois, s'est finalement achevé trois ans plus tard, le premier vol commercial ayant été inauguré le 4 novembre 2002. L'île est désormais desservie deux fois par semaine par avion (jusqu'à trois fois pendant les périodes de vacances scolaires). Le taux de remplissage des premiers mois est de $60 \%$, soit un taux légèrement inférieur à celui de Tubuai avec un prix aller-retour de 37000 FCFP. La réalisation de la piste par remblaiement d'une partie du lagon a employé jusqu'à trente personnes originaires de Raivavae, des Australes et de Tahiti, répondant ainsi à un des premiers objec- tifs assignés à l'aéroport : donner un travail salarié à la population de l'île.

Le coût de l'aéroport de Raivavae est estimé à 1,5 milliard FCFP (La dépêche de Tahiti, 28 septembre 2000), mais d'autres projets ont été réalisés - ou sont en cours de réalisation - parmi lesquels le bétonnage d'une partie de la route de ceinture, la construction d'une route traversière, une marina pour les yachts et les bonitiers, et la rénovation de bâtiments administratifs à commencer par la mairie. Parmi les projets en cours, celui de la distribution de l'eau potable (l'eau à Raivavae est de mauvaise qualité et entraîne un certain nombre de problèmes de gastro-entérites et d'hépatite), a été temporairement abandonné face aux réticences de la population, la solution retenue - par forage - risquant d'assécher les plans d'eau.

La première justification d'un aéroport à Raivavae est d'ordre sanitaire. Il s'agit de sauver des vies en permettant des «évasans 》 (évacuations sanitaires) en cas d'urgence à Papeete, et tous s'accordent aujourd'hui à reconnaître qu'il n'est plus possible de raisonner comme autrefois, où la question des vies sauvées était éminemment religieuse :

« Mon grand-père a dit (lors de l'abandon du premier projet), que les maladies, c'est depuis le temps d'Adam et Ėve, les maladies, ça va pas s'arrêter jusqu'à la fin du monde, il y a toujours des gens qui crèvent, il y a toujours des gens qui meurent, et il faut croire en Dieu, c'est Dieu qui nous soigne. » (Homme né en 1964, parti à Tahiti en 1982 pour le service militaire, habitant à Papara, entretien de décembre 2001)

Mais c'est précisément parce que l'aéroport ne devrait servir qu'aux évasans que certains auraient préféré, soit une liaison aérienne mais non commerciale, soit des hélicoptères de l'armée ou des hydravions. Car l'avion emmène les malades à Papeete, mais risque aussi de ramener des étrangers et des touristes sur l'île. $\mathrm{La}$ première raison suscitant incertitudes et mécontentements d'une partie de la population est directement associée à la transformation des modes de vie consécutive à l'arrivée d'étrangers et de touristes sur l'île, dont on se demande comment les accueillir: comme chez eux ou comme chez nous? Un certain nombre d'interlocuteurs font ainsi valoir qu'avec les touristes, c'est le rythme de vie profondément associé à l'identité raivavae et protestante qui risque d'être remis en cause avec notamment la levée de l'interdiction de vente d'alcool et l'établissement

3. Le remblaiement d'une partie du lagon a accru les cas de ciguatera et a rendu les poissons du lagon impropres à la consommation. 
du courant électrique vingt-quatre heures sur vingt-quatre, y compris le dimanche. Les touristes, indépendamment de leur présence effective, cristallisent toutes les attentions parce qu'ils incarnent toutes les formes de changements susceptibles d'affecter durablement la vie communautaire insulaire.

Alors que les autorités religieuses de l'île de Raivavae, à forte majorité protestante, n'ont jamais caché leur hostilité face à un tel projet, jugé trop coûteux et déstabilisateur du mode de vie traditionnel, la réalisation de ce projet laisse entrevoir la mise en place de nouvelles relations entre les autorités religieuses et politiques. Le fait que le maire ait pu prendre seul cette décision sans l'accord des diacres - qui étaient également jusqu'aux élections de mai 2001 le plus souvent conseillers municipaux - traduit une autonomisation des autorités politiques par rapport aux autorités religieuses initiée par l'Église évangélique de Polynésie française (EEPF). Cette dernière, soucieuse depuis le début des années 1980 de se démarquer officiellement de la politique du territoire et de l'État dans un certain nombre de domaines, à commencer par ceux liés aux essais nucléaires et à leurs retombées (Malogne-Fer, 2003), a ainsi demandé en 2001 aux diacres de choisir entre leurs responsabilités politiques et ecclésiastiques. Désormais le 'āpo'ora'a tāhō'ē, assemblée réunissant mensuellement les diacres des trois paroisses de l'île, n'est plus consultée qu'occasionnellement sur les projets municipaux.

L'exemple de Rapa (497 habitants en 2002), l'île la plus australe de Polynésie française, illustre la façon dont le projet d'aéroport n'engage pas uniquement ceux qui sont restés sur l'île, mais également ceux qui ont émigré à Tahiti. Le projet d'un aéroport, remis à l'ordre du jour suite à la construction de celui de Raivavae, fut l'objet d'une consultation de la population de Rapa sous forme de référendum à l'initiative du maire en janvier 2001. Bien que les Rapa se soient prononcés en faveur de l'aéroport (96 voix pour, 70 contre), la question de l'emplacement destiné à accueillir la piste d'aviation compromet sérieusement la réalisation de ce projet. Surtout, la consultation référendaire a suscité le mécontentement d'une partie des Rapa qui n'ont pas été consultés. Ainsi, Roti Make, originaire de Rapa mais vivant à Tahiti et présidente de l'association Oparo Paruru Ia Rapa, estime que :

« le projet de piste ne se justifie pas et qu'il porterait même gravement atteinte à l'écosystème de Rapa et à son style de vie communautaire si particulier. » (Les Nouvelles de Tahiti, 9 janvier 2001)
Elle souligne surtout que le projet de construction d'un aéroport n'est pas de la compétence de la commune :

« c'est une affaire qui concerne la population de souche rapaienne et donc le conseil de sages. En aucun cas, il ne s'agit d'une compétence territoriale ou d'État » (La Dépêche de Tahiti, 11 avril 2001)

Bruno Saura rappelle que les to'o hitu, juridictions coutumières spécialisées dans les affaires de terres, ont été réactivées dans les années 1980 aux Australes soit sous forme associative (à Rurutu), soit sous forme de commission municipale (à Rapa) (Saura, 1995: 125-128 ; également Malogne-Fer, 2000 : 70-73). Il s'agit moins, en définitive, d'une opposition en termes d'attribution de compétences entre le to'o hitu - représentant les familles de l'île et chargé d'allouer les droits d'usage de cette île non cadastrée - et la municipalité, que d'une volonté d'associer aux projets insulaires des personnes qui ne vivent plus à Rapa mais qui continuent à vouloir revendiquer leurs droits fonciers. La position de Roti Make n'est pas unanimement partagée, un pasteur originaire de Rapa estime au contraire que les gens de Tahiti n'ont pas à enfermer ceux qui sont restés à Rapa :

«Je pense qu'il faudra que les Rapanais prennent une décision favorable, mais les Rapanais d'ici ne vont pas décider pour les gens qui sont restés là-bas. C'est comme quand on retourne là-bas, les Rapa d'ici disent “je veux m'installer là", je ne suis pas d'accord. C'est à ceux qui ont su garder l'île et rester sur l'île de décider ce qu'ils veulent faire. S'ils n'étaient pas sur l'île pour débrousser et faire fructifier les terres, alors on retournerait sur l'île et il n'y aurait plus que de la brousse ou alors l'île serait squattée par d'autres gens. Il faut laisser la décision aux gens de l'île. » (Homme né au début des années 1950, parti à Tahiti pour les études, puis pour la Nouvelle-Calédonie pour le travail, pasteur de Rapa de 1986 à 1989, entretien de décembre 2000)

\section{Les Raivavae émigrés à Tahiti : organisation paroissiale, mémoire et identité}

Il n'existe pas de données précises disponibles sur le nombre de Raivavae émigrés à Tahiti, notons simplement que les Raivavae de Tahiti sont plus nombreux que ceux restés au fenua (terre d'origine). L'archipel des Australes est le seul qui a vu sa population décroître entre 1996 et 2002 (ISTAT, 2003a : 3). L'exode rural touche différemment les îles de cet archipel : alors que Rurutu et Tubuai - dotées d'aéroports depuis les années 1970 - ont vu leur population augmenter, celle des îles accessibles seulement par bateau a 
diminué. C'est à Raivavae que la dépopulation est la plus manifeste (19\% entre 1988 et 2002), la migration des Raivavae à Tahiti est essentiellement le fait des hommes et femmes en âge de travailler (entre 20 et 50 ans), contribuant au vieillissement de la population restée sur l'île ${ }^{4}$. Fait nouveau, les départs semblent définitifs, comme l'atteste le nombre de logements vacants à Raivavae : pratiquement un logement sur quatre (23\%) (ISTAT, 1996 : 157).

Il n'existe pas à notre connaissance d'études sur les populations émigrées à Papeete et sur la façon dont elles s'organisent et vivent leur exil et leur intégration en ville. Christine Jourdan rappelle que le peu d'études anthropologiques consacrées aux recompositions des relations sociales en milieu urbain dans le Pacifique provient de difficultés méthodologiques liées à l'absence d'entités nettement circonscrites, mais surtout au contexte historique de développement de l'anthropologie qui :

« met l'accent sur l'exotique, le primitif, l'authentique, et si possible, l'inconnu [...] Curieusement, alors que les populations du Pacifique semblent être fascinées par le monde urbain, les anthropologues, à quelques exceptions près, ne semblent pas être intéressés par les nouvelles formes de relations sociales que l'on y trouve. » (Jourdan, 1994 : 177-179)

Or, l'étude des populations émigrées ouvre des perspectives intéressantes pour la compréhension des transformations sociales. Les personnes originaires des Australes émigrées à Tahiti continuent pour la plupart (au moins pour la première génération) à entretenir des relations entre elles grâce au cadre paroissial, rendant ainsi l'étude anthropologique méthodologiquement possible. L'analyse de la façon dont les émigrés envisagent - avec réticence - le projet d'un aéroport à Raivavae souligne l'importance du concept de mémoire dans le processus d'acceptation et de relativisation des transformations des modes de vie à Tahiti.

À l'exception des deux paroisses de langues française (Béthel) et chinoise (Jourdain) de Papeete, toutes les paroisses de L'EEPF se divisent en plusieurs 'āmuira'a ou groupes, chaque åāmuira'a regroupant au sein d'une même paroisse les personnes selon leur origine géographique et/ou familiale (Vernier, 1986: 102 et 362). En milieu urbain, la dissociation entre lieu d'habitation et lieu d'origine rend la compréhension des découpages en 'ämuira'a plus complexe dans la mesure où les 'ämuira'a regroupent désormais des personnes qui n'ont plus forcément de liens de parenté ni même de voisinage entre elles. L'identité des migrants est elle-même sans cesse requestionnée et retravaillée. Les gens originaires des Australes mais vivant à Tahiti ne sont plus complètement considérés comme des tuha'a pae, ils ne sont pas pour autant tahitiens. Une femme originaire de Rapa qui a quitté l'île à l'âge de cinq ans dit ainsi :

« tu as en face de toi une Rapa ratée. »

Une jeune fille originaire de Raivavae qui a quitté l'île à onze ans pour poursuivre ses études à Tubuai puis à Tahiti a longtemps hésité avant de confirmer son baptême à Tahiti (et non à Raivavae) parce qu'elle avait «toujours la tête là-bas »; à Tahiti, on lui fait remarquer qu'elle parle avec l'accent raivavae ; à Raivavae qu'elle n'a pas grandi au fenua. Cette situation parfois inconfortable s'accompagne le plus souvent d'une double appartenance paroissiale : arrivés en ville, le souci des migrants ou des parents est de trouver une paroisse d'accueil pour continuer à aller au culte mais surtout pour s'assurer, qu'en cas d'accident, une paroisse prendra en charge l'organisation des funérailles et éventuellement le rapatriement du corps.

« Après Rurutu, je suis venu ici pour mes études, j’ai perdu un peu la notion d'école du dimanche, du purera'a, je n'allais pas, quand j'étais en seconde. C'était aussi peut-être le fait qu'ici à Tahiti je découvre beaucoup de choses, les camarades et, petit à petit, je me suis dit "je dévie", je change peut-être de chemin. Dans ma tête, si j'ai un accident ici, je meurs, où on va m'emmener ? dans quelle paroisse ? Et après j'ai continué à aller à Peteteta la communauté rimatara à Faripitii, l' 'âmuira'a de Tiroama. C'est par là que je suis allé au purera'a, à un moment donné, ils m'ont demandé d'être moniteur de l'école du dimanche, je n'ai pas refusé. » (Homme né au début des années 1970, originaire de Rimatara, parti pour les études au collège à Rurutu puis au lycée à Tahiti, habitant Papeete)

Il ne s'agit pas pour autant d'abandonner sa paroisse d'origine et nombreux sont ceux qui continuent à lui envoyer de l'argent pour signifier qu'ils en font toujours partie et rappeler qu'il ne faut pas les oublier.

La particularité des migrants originaires des Australes est de continuer à s'organiser par origine insulaire alors même que les retours au fenua, définitifs ou temporaires, sont relativement rares (Lockwood, 1990). La grande

4. La tranche d'âge comprise entre 20 et 49 ans représente $34,4 \%$ de la population à Raivavae, $38,9 \%$ à Tubuai et $45,2 \%$ à Tahiti. Les personnes âgées de 50 ans et plus représentent $17,4 \%$ de la population à Raivavae, $15,4 \%$ à Tubuai et $12,8 \%$ à Tahiti (ISTAT, 1996 : 120-125). 
paroisse de Tiroama à Papeete est ainsi organisée en sept 'ámuira'a dont trois sont exclusivement réservés aux personnes originaires des Australes : les Raivavae et Rapa se retrouvent dans le 'àmuira'a Taroma, les Rurutu dans le 'āmuira'a Ebene Etera et les Rimatara dans le 'āmuira'a Peteteta, les trois fare 'ämuira'a (les maisons de groupes) sont situés dans le quartier Puea de Papeete. À Tahiti, les paroisses de Faa'a, Papara et plus récemment de Paea et Mahina comportent également en leur sein un ou plusieurs 'àmuira'a regroupant les gens originaires des Australes. L'appartenance au 'ämuira'a est plus forte que celle paroissiale dans la mesure où elle permet d'associer pratique religieuse et liens communautaires :

"Quand on arrive des îles, on pense que le fare 'àmuira' a peut nous donner beaucoup de choses, on ne parle pas seulement de la spiritualité, on parle aussi de la vie de tous les jours, on essaie d'améliorer l'ordinaire de la vie quotidienne. » (Pasteur de Tahiti originaire de Rapa, décembre 2000)

L'organisation paroissiale permet une telle association, puisqu'à l'exception du culte dominical, la majeure partie des activités (école du dimanche, activités des jeunes, des femmes, tuāro' $i$, préparation des chants) continuent d'être organisées au niveau de chaque 'ämuira'a. Les chants qui sont propres à chaque île sont l'occasion de se démarquer des autres 'āmuira'a et sont fréquemment invoqués pour expliquer le regroupement des Australes au sein de la paroisse et la création d'autant de 'àmuira' a qu'il y a d'îles. Le fare 'āmuira'a accueille ainsi répétition de chants, repas de mariages, rencontres sportives et veillées funéraires. À chaque rencontre, il est toujours possible de manger du taro de Raivavae ou du poisson de Rapa, envoyés par ceux qui sont restés au fenua et conservés dans les congélateurs du fare 'àmuira'a.

La concentration sur Papeete des structures hospitalières oblige les malades des îles à émigrer pour des séjours plus ou moins longs à Papeete. Les communautés des Australes installées à Tahiti sont dès lors chargées de visiter les malades et veiller les morts.

«À Rapa, on voit par famille, tandis qu'ici, on dirait on est une seule famille, on est ensemble, même si on ne vit pas dans un même district, c'est tout le monde, éparpillé, mais quand on vient se rassembler, c'est tout le monde, c'est une famille unie. C'est comme ça qu'on vit. S'il y a un décès rapa, c'est toute la famille réunie. Ici dans le 'àmuira'a, il y a des enfants raivavae et des enfants rapa. Quand il y a un décès de Rapa ici, tous les Rapa viennent. » (Diacre du 'àmuira'a RaivavaeRapa, paroisse de Tiroama)
Les morts sont veillés par la communauté installée à Tahiti et le plus souvent rapatriés et enterrés sur l'île d'origine. L'organisation paroissiale en 'āmuira'a permet ainsi aux migrants installés à Tahiti d'entretenir des liens entre eux mais aussi avec ceux qui sont restés aux Australes. Une des motivations avancées pour justifier la création d'un nouvel 'āmuira'a et, ce faisant, la construction d'un fare 'àmuira'a, est précisément de pouvoir accueillir les paroissiens des Australes lorsqu'ils se déplacent à Tahiti, le plus souvent pour participer à des manifestations religieuses (la commémoration de l'arrivée de l'évangile le 5 mars qui est jour férié en Polynésie française) ou des manifestations culturelles (les concours de danses de chants et d'artisanat qui ont lieu en juillet lors du heiva).

À travers la situation des migrants, l'identité est requestionnée. Elle reste liée à la terre, la langue et la nourriture, mais le lien sans être rompu s'est quelque peu distendu, l'essentiel n'est plus de cultiver directement la terre familiale mais plus simplement de faire en sorte que la terre familiale reste entretenue par un membre de la famille (l'émigration à Tahiti n'empêche pas de cultiver un jardin locatif ou plus rarement de devenir propriétaire et de planter des pieds de 'uru venus de Raivavae ou des arbres fruitiers). L'identité se définit aussi par un ensemble de relations sociales et paroissiales, par la permanence de projets communs qui sont autant d'occasion de «travailler» ensemble pour reprendre l'expression d'un diacre du 'āmuira'a Raivavae-Rapa :

«On a les liens à travers la terre en premier, en deuxième, c'est à travers le travail, le plus grand lien, c'est entre la population rapa qui est sur la terre rapa et les enfants rapa qui sont sur la terre Tahiti. »

L'ouverture d'une piste d'aviation engendre de fait une reconfiguration des relations entre les insulaires et les Raivavae émigrés à Tahiti, elle interroge ces derniers sur leur exil et leur propre conception de la modernité, associée à la vie salariée et aux échanges monétarisés, qu'ils acceptent en venant sur Tahiti mais qu'ils refusent de voir introduits à Raivavae.

L'ouverture d'une piste d'aéroport à Raivavae oblige les insulaires émigrés à Tahiti à s'interroger sur les raisons de leur exil prolongé et, ce faisant, sur un éventuel retour. Les premières migrations des gens des Australes à Tahiti (mais aussi à Hao et Moruroa) sont d'abord motivées par des raisons économiques et associées au développement de l'emploi salarié consécutif à l'implantation du CEP au début des années 1960 
(Rallu, 1997 : 31). Il s'agissait le plus souvent d'une migration masculine et, en principe, temporaire. Les Raivavae habitant aujourd'hui à Tahiti invoquent au contraire une multitude de raisons et un effet d'entraînement : il a fallu tout à la fois trouver un travail, rester pour la scolarité des enfants et la santé des parents. Si le retour n'est jamais immédiatement envisagé et planifié, il n'est pas non plus complètement écarté et nombreux sont ceux qui ont une maison au fenua - construite ou en projet - pour leur retraite, leurs vacances ou les enfants. Deux exemples illustrent la multiplicité des raisons qui empêchent ou retardent un retour au fenua.

T. est né en 1938, il est originaire de Raivavae et est parti avec ses parents à Tahiti en 1942 :

«Quand on arrive à Papeete pour la première fois, c'était déjà beaucoup pour nous, on croyait que les voitures, c'était comme une bête qui allait nous dévorer. »

Il se souvient de sa période adolescente à Papeete dans les boîtes de nuit avant de partir en 1962 pour Rapa comme instituteur. Aujourd'hui, il est marié avec une femme originaire de Rapa, vit entre Rapa et Papara (Tahiti, zone rurale), dans un quartier habité en majorité par des gens originaires des Australes et s'interroge sur un éventuel retour à Rapa :

« le plus jeune (des enfants) est au collège de Papara. Quand il aura fini sa scolarité, c'est selon la santé qu'il y aura retour ou non sur Rapa. » (Entretien, octobre 2000)

T. Vahine est originaire de Raivavae, son mari est diacre du 'ammira'a Ravaivae-Rapa de la paroisse de Tiroama à Papeete. Elle part à Tahiti en 1966 :

« moi j’ai amené une petite-nièce à l'hôpital, c'est comme ça que je suis venue. »

C'est à Tahiti qu'elle rencontre son mari, originaire de Rapa :

« lui est là pour le travail, il a commencé à Moruroa de 1966 à mars 1968 [...]. Lui, il a continué comme travail en 1968 à Total d'abord, sur le quai comme docker, et après chez Chevron, à partir d'août 68 et pendant trente-et-un ans $»$.

Ils envisagent de prendre leur retraite à Raivavae mais refusent de renoncer pour l'instant aux responsabilités assumées depuis vingt ans dans le 'āmuira'a :

« Pour notre retraite, on a commencé à construire à Raivavae. On est resté à Tahiti parce qu'il avait un bon travail, il a bien continué. Avec les enfants qu'on a eus, après avec l'école, c'était pas facile non plus notre vie ; on est diacres, c'est ça qui nous a bien retenus, on est retraités du travail mais pas retraités de diacre. On est diacres depuis 1978, avril 1978. " (Entretien, janvier 2001)

À travers les récits des migrants, la recherche d'un(e) époux(se), sans être jamais évoquée comme cause première de départ, apparaît déterminante pour expliquer l'ampleur de l'exode à Tahiti et la faiblesse des retours. Les liens familiaux sont tels qu'il est en effet difficile de se marier avec une personne originaire de Raivavae avec laquelle on n'a pas de liens de parenté (l'interdiction de mariage entre fêt $t$ ' $i$ - parents s'applique en principe jusqu'à la quatrième génération). Les Raivavae de Tahiti ont le plus souvent épousé une personne originaire d'une autre île des Australes (Rapa, Rurutu et Rimatara) apportant la preuve de l'efficacité du cadre paroissial et tout particulièrement des activités de l'Union chrétienne des jeunes gens (UCJG) en termes de socialisation des jeunes.

L'aéroport a une première conséquence directe pour les Raivavae à Tahiti, celle de pouvoir désormais passer les congés à Raivavae sans « finir les congés sur le bateau ». Mais, le prix du billet d'avion risque de freiner les allers-retours, surtout s'ils s'effectuent en famille. Une seconde option est alors vite envisagée, celle de faire venir pendant les dites vacances le ou les parents restés à Raivavae, confortant par là tous ceux qui pensent que l'aéroport ne va pas enrayer l'exode rural mais au contraire l'accélérer.

L'ouverture de la piste d'aviation met en avant une conception de la modernité associée à la vie salariée et aux échanges monétarisés que les migrants acceptent en venant à Tahiti mais qu'ils refusent de voir introduits à Raivavae. Les Raivavae de Tahiti, lorsqu'ils évoquent l'aéroport et ses conséquences attendues, insistent avant tout sur les changements, irréversibles et redoutés, associés à l'arrivée de l'argent. Avec l'aéroport, les gens vont devenir individualistes, ils vont se détourner des fa'a'apu (plantations) et travailler pour un patron au lieu de travailler pour euxmêmes :

« Déjà, c'est le problème au niveau de la coutume, les gens ne vont plus faire le $f a^{\prime} a^{\prime} a p u$, ils pensent tout le temps à l'argent. »

Tous s'accordent à dire que contrairement à Tahiti où tout s'achète, il est possible de vivre avec peu d'argent à Raivavae et de vivre bien, ce que résume le maire de Raivavae en ces termes:

« la vie à Raivavae, c'est facile, c'est pas comme ici à Tahiti, à Tahiti tu as de l'argent tu peux vivre, mais à 
Raivavae, même si tu n'a pas d'argent, tu peux vivre seulement (quand même). »

La description est celle d'une vie idéalisée qui n'existe déjà plus mais qui dans le même temps continue de servir de cadre de référence pour tous ceux qui vivent à Tahiti. La transformation des modes de vie à Tahiti (urbanisation, salariat) était acceptée dans la mesure où elles ne remettaient pas en cause l'essentiel : l'identité raivavae, le rapport à la terre et à l'origine. Les transformations attendues à Raivavae sont d'autant plus redoutées qu'elles affectent le paysage communautaire au sein duquel les migrants ont grandi et qu'elles portent atteinte aux « cadres sociaux de la mémoire » pour reprendre une notion développée par Maurice Halbwachs :

«Sociétés religieuses, politiques, économiques, familiales, groupes d'amis, de relations et même réunions éphémères dans un salon, dans une salle de spectacle, dans la rue, toutes immobilisent le temps à leur manière, ou imposent à leurs membres l'illusion que pendant une certaine durée tout au moins, dans un monde qui change sans cesse, certaines zones ont acquis une stabilité et un équilibre relatifs et que rien d'essentiel ne s'y est transformé pendant une période plus ou moins longue. » (1997 : 192)

Analysant la façon dont la mémoire collective se construit, Maurice Halbwachs souligne l'importance du temps mais aussi de l'espace dans le processus de mémorisation :

« la mémoire collective prend son point d'appui sur des images spatiales [...] Tel habitant pour qui ces vieux murs, ces maisons décrépies, ces passages obscurs et ces impasses détruits faisaient partie de son petit univers, et dont bien des souvenirs se rattachent à ces images maintenant à jamais effacées, sent que toute une partie de lui-même est morte avec ces choses. » (1997 : 200-201)

Cette mémoire raivavae est d'autant plus vive à Tahiti que le fonctionnement paroissial en 'āmuira' $a$ associe étroitement mémoire religieuse et mémoire communautaire. Et lorsque les migrants retournent en groupe à Raivavae ou à Rapa c'est le plus souvent pour participer à l'inauguration d'un temple ou d'un fare 'ämui$r a^{\prime} a$ auquel ils ont financièrement participé.

« Pour le temple de Ahurei, les Rapa de Tahiti ont donné 9 millions de francs, c'est à peu près la même part que les Rapa de Rapa. Ils ont moins de dépenses, le sable, le gravier, c'est là-bas. C'est à nous les fenêtres, les portes, c'est aux enfants d'ici, c'est nous qui avons pris la dépense, et aussi les carreaux et la sono. » (T. Vahine).

À chacune de ces inaugurations ou des commémorations religieuses, les émigrés et leurs parents restés au fenua se retrouvent au sein de l'espace religieux autour d'une représentation idéale de l'identité raivavae : il s'agit de se prouver, à soi et aux autres, que l'essentiel est sauf, à travers la mise en scène de la permanence d'une identité raivavae indissociable de la foi protestante.

Si les Raivavae émigrés à Tahiti continuent à regretter la construction d'une piste d'aviation à Raivavae alors que ceux restés au fenua se sont progressivement ralliés au projet du maire, c'est parce que dans le cas des migrants, l'aéroport touche à l'essentiel, l'origine et l'identité raivavae. Ce faisant, les Raivavae n'expliquent pas pourquoi ils ont émigré alors qu'à Raivavae on vit avec peu et on vit bien. Une des raisons non avouées, de départ ou de non-retour, est que la vie à Tahiti permet de se soustraire au contrôle familial et communautaire parfois jugé étouffant. Les départs de l'île constituent une modalité, parmi d'autres, de régulation des tensions familiales (notamment à propos des affaires de terres). Une femme diacre, à l'origine de la création d'un 'ämuira'a des Australes dans la paroisse de Paea (Tahiti) découvre avec une certaine amertume que «vivre parmi nous, c'est insupportable!» :

«Et là je vis avec toutes les Australes et dans ma tête tout au début quand on a fondé cet 'àmuira' $a$, dans ma tête, tout au début, ce sont des gens qui accueillent plus facilement, ce sont des gens qui ne regardent pas les problèmes, au contraire ils vont vers les problèmes pour aider les gens. Et quand ce 'āmuira'a s'est fondé, je disais à mon mari, je me suis trompée sur ces personnes-là, parce qu'on ne vivait pas ensemble, là aujourd'hui on vit vraiment ensemble [...]. Et je trouve aujourd'hui, vivre parmi nous, c'est insupportable ! »

Les migrants refusent l'aéroport qui signifie l'arrivée de l'argent et des touristes à Raivavae, ils craignent que les Raivavae se détournent des plantations pour mieux se lancer dans les activités touristiques et salariées. Pourtant, ils sont les premiers à avoir initié cette patrimonialisation de l'île en vivant hors de l'île tout en revendiquant une part d'héritage et en confiant à un parent proche le soin d'entretenir la maison laissée vacante et de défricher les terres inexploitées. Il existe de fait une certaine affinité entre les migrants qui souhaitent une île protégée de façon à ce qu'elle reste conforme aux souvenirs et les touristes qui ont à cœur de retrouver des paysages préservés, à la différence près que, dans le second cas, les touristes payent cher pour venir en Polynésie française confortant par là la position de ceux qui veulent garder le mode de vie traditionnel : 
« Pour moi, les touriste, ils veulent aller là où il y a des coins comme Raivavae, là où ça n'existera plus. Mêmes nous, on veut des coins tranquilles, il n'y a pas que les touristes qui pensent comme ça! Le fenua qu'on a maintenant, il faut le garder. À travers ceux des îles, ils disent, ça leur fait penser cet endroit calme, la mer, tout ça c'est chez nous ! et vous voulez changer ? Une fois changer, où vous allez aller ? Les touristes dépensent beaucoup juste pour voir un truc : le sable blanc, la mer bien propre, tout ça c'est chez nous, il faut pas qu'on devienne comme eux (comme les touristes à la recherche d'un endroit calme et paisible). » (Homme né en 1971, venu à Tahiti pour les études, marié à une Rimatara)

C'est bien le rapport à l'argent qui constitue la pierre d'achoppement des initiatives de développement dans les archipels éloignés. Alors que l'argent est nécessaire pour acheter des denrées au magasin et acquérir des biens de consommation, la possibilité d'en gagner autrement qu'en travaillant grâce aux nombreuses subventions destinées aux "porteurs de projets » oblige à repenser les mécanismes de rentabilité et de réussite économiques selon une logique comparable à celle de Nouvelle-Calédonie :

« les conditions de fonctionnement de l'économie calédonienne découragent l'effort productif. Ainsi, l'action pour stimuler le "développement" reste irrémédiablement contrariée par les structures macroéconomiques d'un système qui, tel un tonneau des Danaïdes, tire de l'assistance la perpétuation de sa dépendance. » (Bensa et Freyss, $1994: 25$ )

Les subventions allouées permettent, grâce à un apport initial, de donner vie à un projet, mais elles prouvent aussi une bonne connaissance des circuits et des rouages administratifs et ce faisant signifient une bonne insertion sociale. Victoria Lockwood rappelle très justement que la pauvreté est d'abord une pauvreté sociale et morale : elle affecte ceux chez qui les liens traditionnels de solidarité familiale et communautaire ne fonctionnent plus (Lockwood, 2002). De la même façon, parce que la richesse est d'abord sociale, l'aéroport à Raivavae signifie moins un possible développement économique de l'île que la preuve visuelle et matérielle de la bonne intégration de l'île dans l'espace politique polynésien.

\section{BIBLIOGRAPHIE}

Al Wardi Sémir, 1998. Tahiti et la France, le partage du pouvoir, Paris, l'Harmattan.

BENSA Alban et Jean Freyss, 1994. La société kanak est-elle soluble dans l'argent... ?, Terrain 23, pp. 1126.
FER Yannick, 2000. Coopérative, familles et développement en Polynésie française : la coopérative de pêcheurs Piareare, Rurutu (archipel des Australes), Revue juridique polynésienne 6, pp 527-536.

Fer Yannick et Gwendoline Malogne-Fer, 2000. Tuaro’i, réflexions bibliques à Rapa, Papeete, éd. Haere po.

Guillebaud Jean-Claude, 1980. Un voyage en Océanie, Paris, éditions du Seuil.

Halbwachs Maurice, 1994 ( 1 ère éd. : 1925). Les cadres sociaux de la mémoire, Paris, Albin Michel.

—, 1997 (1 $1^{\text {ère }}$ éd. : 1950). La mémoire collective, Paris, Albin Michel.

Institut DE LA STATISTIQUe DE LA Polynésie FRANÇAISE (ISTAT), 1999. Résultats du recensement général de la population de la Polynésie française du 3 septembre 1996, Papeete.

—, 2003a, Points forts 4, Papeete.

—, 2003b, Étude Budget des familles, 2000-2001, principaux résultats, Papeete.

JoURDAN Christine, 1994. Créolisation, urbanisation et identité aux îles Salomon, Journal de la Société des Océanistes 99, pp. 177-185.

LA DÉPECHE DE TAHITI, 2000 (28 septembre). Marina et piste d'aviation : les grands travaux en photos.

—, 2001 (11 avril). Vers l'annulation du référendum de Rapa.

LES Nouvelles de TAHiti, 2001 (9 janvier). Piste d'aviation à Rapa, la population vote pour le "oui".

Lockwood Victoria, 2002. Poverty in Paradise: Development and relative income pverty in rural Tahitian society, Human Organization 61, 3, pp. 210225.

-, 1990. Developpement and return migration to rural french polynesia, International Review 24, 2, pp. 347-371.

MaloGne-Fer Gwendoline, 2003. L'église évangélique de Polynésie française et les essais nucléaires : de la prise de conscience chrétienne à la prise de position publique, 1963-1982, François Mitterrand et les territoires français du Pacifique (1981-1988), Jean-Marc Regnault (eds), Paris, Les Indes savantes, pp. 205-214.

MinistèRe DES ARCHIPELs, 1998. Îles Australes, recueil des données essentielles, Papeete.

PANOFF Michel, 1970. La terre et l'organisation sociale en Polynésie. Paris, Payot.

POIRINE Bernard, 2002. L'économie de "l'après-CEP" : forces et faiblesse, La France et les outre-mers, l'enjeu multiculturel, Hermès 32-33, pp. 317-333.

Rallu Jean-Louis, 1997. Population, migration, développement dans le Pacifique Sud, Paris, UNESCO.

SAura Bruno, 1995. Les règles coutumières en Polynésie française, in Paul de Deckker (éd.), Coutume autochtone et évolution du droit dans le Pacifique Sud, Paris, L'Harmattan, pp. 95-131.

Vernier Henri, 1986. Au vent des cyclones, Papeete, éd. Haere po no Tahiti. 